\title{
Simulation of Employee Behavior Based on Cellular Automata Model*
}

\author{
Yue Jiao ${ }^{1}$, Shaorong Sun ${ }^{1}$, and Xiaodong $\operatorname{Sun}^{2}$ \\ ${ }^{1}$ College of Management, University of Shanghai for Science and Technology, Shanghai \\ 200093, P.R. China \\ ${ }^{2}$ Antai College of Economics and Management, Shanghai Jiao Tong University, Shanghai \\ 200052, P.R. China \\ jiaomooner@hotmail.com, sunshaorong@usst.edu.cn, \\ mlshmily@sjtu.edu.cn
}

\begin{abstract}
The aim of this current paper is to research the interactive influence of employee behavior in a given organization. First, we define three kinds of employee behavior called Positive Behavior, Zero Behavior and Negative Behavior. Then, we give a new cellular description of behavior states and define the evolution rules for this cellular automata (CA) model. In order to find what may influence the employee behavior and how, we consider two cellular attributes: behavior's influence force, recoded as Influence and behavior's insistence force, recorded as Insistence. Finally, we use this improved CA model to simulate how employee behavior evolves, and how encouragement rules and punishment rules influence employee behavior.
\end{abstract}

\section{Introduction}

Employee behavior is the action of attitudes, working style, planning directly or indirectly evoked in the work. Active and energetic behavior is good for the organization to obtain their goals, and vice versa. Analyzing the employee behavior is useful for managers to lead negative behavior to positive side [1]. It is difficult to describe and look into the interactive influence among employees by applying general mathematical models. However, the self-reproducing and neighborhood rule of CA are very suitable for simulating employees and their behaviors in an organization, for they affect their neighbors' behavior and are affected by their neighbors and this process is a complex self-reproducing.

Cellular automata are simple models of computation which exhibit fascinatingly complex behavior. They have captured the attention of several generations of researchers, leading to an extensive body of work [2]. To some extent, CA can be used to reflect the behavior of human. So we apply CA to simulate employee behavior in a given organization in order to analysis how employee behavior evolves, and how encouragement rules and punishment rules influence employee behavior.

\footnotetext{
* Supported by the Program of National Natural Science Foundation of China, No. 70271005, No. 70471066; Supported by the Program of Shanghai Important Basic Research Program, No. 03JC14054; Supported by Shanghai Leading Academic Discipline Project, No.T0502.
} 


\section{Cellular Automata Model for Employee Behavior}

Employee behavior which is encouraged and propitious to the management, production, creation and cooperation of the organization, could be called Positive Behavior(PB), such as invention of technology, retrenchment of resource; employee behavior which is the behavior not encouraged, forbidden by the rule or the culture of the organization, could be called Negative Behavior(NB), such as privilege abuse and theft $[3,6]$; and Zero Behavior(ZB), between Positive and Negative, is neither encouraged nor forbidden. $\mathrm{ZB}$ may be the leak of the rule, or is not heavy to such an extent that the object can be punished, such as absenteeism, substance abuse [4]; it may be inaction, such as do-nothing behavior.

Employees in the organization are looked as the CA space, and each employee is a cell. Distance between cells is not the distance in physics, but in psychology and behavior. Each cell is influenced by its neighbors, and at the same time influences others, which cause the evolution and the update of the employee behavior. The closer the distance is, the more influence, and vice versa.

In CA space every cell has three behavior states $S_{i, j}^{t}=\{1,0,-1\}$, where 1 is the PB, 0 is the $\mathrm{ZB}$ and -1 is the NB. For the employee in the organization, everyone has his difference. We think about two characteristics related to employee behavior: Influence and Insistence. Influence is the extent that some employee affects his neighbors. Insistence is the extent of the employee's holding his own behavior. High-Insistence employee is difficult affected by his neighbors [2]. So, each cell has two characteristics: Influence $I N F_{i, j}=\{1,2,3\}$, Insistence $I N S_{i, j}=\{1,2,3\}$, where each characteristic has three degrees as we hypothesis.

Employee behavior is affected by his neighbors. Different neighbor behavior makes different influence to the cell. The cumulate influences of $\mathrm{PB}, \mathrm{NB}$, and $\mathrm{ZB}$ neighbors on one given cell are separately called Positive, Negative, and Zero Environmental Disturbances Degree, formulated by:

$$
\begin{aligned}
\operatorname{ped}_{i, j}^{t} & =\sum_{i=i-2}^{i+2} \sum_{j=j-2}^{j+2} \frac{I N F_{i^{\prime}, j^{\prime}}}{\sqrt{\left(i^{\prime}-i\right)^{2}+\left(j^{\prime}-j\right)^{2}}}, S_{i^{\prime}, j^{\prime}}^{t}=1 \\
n e d_{i, j}^{t} & =\sum_{i=i-2}^{i+2} \sum_{j=j-2}^{j+2} \frac{I N F_{i^{\prime}, j^{\prime}}}{\sqrt{\left(i^{\prime}-i\right)^{2}+\left(j^{\prime}-j\right)^{2}}}, S_{i^{\prime}, j^{\prime}}^{t}=-1 \\
\operatorname{zed}_{i, j}^{t} & =\sum_{i=i-2}^{i+2} \sum_{j=j-2}^{j+2} \frac{I N F_{i^{\prime}, j^{\prime}}}{\sqrt{\left(i^{\prime}-i\right)^{2}+\left(j^{\prime}-j\right)^{2}}}, S_{i^{\prime}, j^{\prime}}^{t}=0
\end{aligned}
$$

We define local rules:

(1) When $S_{i, j}^{t}=1$, if ped $+I N S_{i, j}=\max \left\{\right.$ ped $+I N S_{i, j}$, ned, zed $\}$, then $S_{i, j}^{t+1}=1$; else, if ned>zed, then $S_{i, j}^{t+1}=-1$; if zed $>$ ned, then $S_{i, j}^{t+1}=0$; if zed=ned, then $P\left\{S_{i, j}^{t+1}=0\right\}=0.5, P\left\{S_{i, j}^{t+1}=-1\right\}=0.5$. 
(2) when $S_{i, j}^{t}=-1$, if ned $+I N S_{i, j}=\max \left\{\right.$ ned $+I N S_{i, j}$, ped, zed $\}$, then $S_{i, j}^{t+1}=-1$; else, if ped $>$ zed, then $S_{i, j}^{t+1}=1$; if zed > ped, then $S_{i, j}^{t+1}=0$; if ped $=z e d$, then $P\left\{S_{i, j}^{t+1}=1\right\}=0.5, P\left\{S_{i, j}^{t+1}=0\right\}=0.5$.

(3) When $S_{i, j}^{t}=0$, if zed $+I N S_{i, j}=\max \left\{z e d+I N S_{i, j}\right.$, ped,ned $\}$, then $S_{i, j}^{t+1}=0$; else, if ped $>$ ned, then $S_{i, j}^{t+1}=1$; if ned $>$ ped, then $S_{i, j}^{t+1}=-1$; if ped $=$ ned, then $P\left\{S_{i, j}^{t+1}=1\right\}=0.5, P\left\{S_{i, j}^{t+1}=-1\right\}=0.5$.

In the evolution process of employee behavior, the policy of the organization plays an important role [5]. It is in that employee will strengthen his behavior intensity when policy encourages the relative behavior, and reduces when forbids. In order to find how the policy affects the employee behavior, we propose the rule:

When the organization encourages the PB of employee,

$$
\operatorname{ped}_{i, j}^{t}=\sum_{i=i-2}^{i+2} \sum_{j=j-2}^{j+2} \frac{\alpha I N F_{i^{\prime}, j^{\prime}}}{\sqrt{\left(i^{\prime}-i\right)^{2}+\left(j^{\prime}-j\right)^{2}}}, S_{i^{\prime}, j^{\prime}}^{t}=1
$$

When the organization punishes the NB of employee,

$$
n e d_{i, j}^{t}=\sum_{i=i-2}^{i+2} \sum_{j=j-2}^{j+2} \frac{\beta I N F_{i^{\prime}, j^{\prime}}}{\sqrt{\left(i^{\prime}-i\right)^{2}+\left(j^{\prime}-j\right)^{2}}}, S_{i^{\prime}, j^{\prime}}^{t}=-1
$$

where $\alpha \in R, \alpha>1$, and $\beta \in R, 0<\beta<1$.

\section{CA Simulation}

The evolution of the employee behavior in an enterprise of $100 \times 100=10,000$ is simulated. The proportion of $\mathrm{PB}, \mathrm{ZB}$ and NB employee and the encouragement and punishment policy is analyzed, and also their effect to the evolution of the employee behavior. The Influence and Insistence is the integer distributing uniformly in $[1,3]$. No encouragement and punishment policy. We simulate: (a) the proportion of $\mathrm{PB}, \mathrm{ZB}$ and NB employee is $P S: Z S: N S=1: 1: 1$, distributing uniformly. The stable state of this situation is shown in Fig.1. The color in figure-black, gray and white is respectively the employee of $\mathrm{PB}, \mathrm{ZB}$ and $\mathrm{NB}$; (b) encouragement policy is put in force to encourage the $\mathrm{PB}$, with $\alpha=1.1$; (c) punishment policy is put in force to punish the

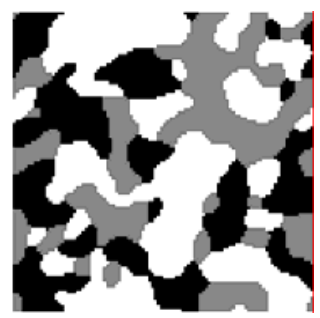

Fig. 1. Stable State

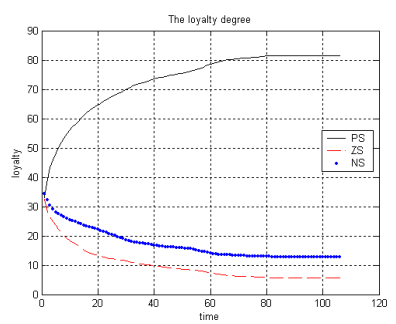

Fig. 2. Employee Proportion

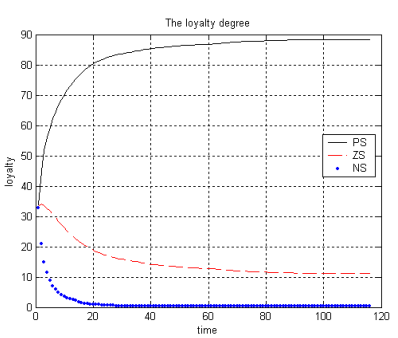

Fig. 3. Employee Proportion 
$\mathrm{NB}$, with $\beta=0.9$; (d) both are put in force at the same time, with $\alpha=1.1, \beta=0.9$. We just give the proportion pictures (shown in Fig.2-3) of situation (b) and (d) because of the length limit of our paper.

Comparing the employee proportion graph of (b), (c), (d) and (a), we find the proportion of PB employee is higher than that in (a) because of the policy. The proportion of $\mathrm{PB}$ employee in (b) is $81 \%$, which is much higher than that in (c), $47 \%$; the proportion of $\mathrm{ZB}$ employee in $(\mathrm{d})$ is $6 \%$, which is much lower than that in (c), 46\%; the proportion of NB employee in (b) is $13 \%$, which is higher than that in (c), $7.5 \%$. The reason is that the encouragement policy builds a hortative environment to reform behavior from Zero and Negative to Positive, while the punishment policy restricts the NB. The transfer from ZB to NB in punishment policy is merely restricted, and to positive one is not encouraged, so the proportion of $\mathrm{ZB}$ employee in (c) is even higher than that in (a). In (d), the proportion of PB employee is the highest, and the proportion of NB employee is the lowest. And the graph (d) changed the most quickly, in that the both policies strengthen the choice of the employee.

Our results reveal that both policies increase the proportion of PB employee, so it is necessary to make relative policies. From the simulation we find that each policy has different effect on different behaviors. In order to reduce the extra cost of the $\mathrm{ZB}$, encouragement policy is better than punishment policy. But to reduce NB, the latter one is more efficient. To increase $\mathrm{PB}$ and reduce NB, the policies may be used together, but there may be no exact effect on controlling $\mathrm{ZB}$.

\section{Conclusion}

In this paper we propose the conception of $\mathrm{PB}, \mathrm{ZB}$ and $\mathrm{NB}$, and research the interactive influence of employees' behavior in a given organization. In order to find what may influence the employee behavior and how, we consider two cellular attributes: behavior's influence force, recoded as Influence and behavior's insistence force, recorded as Insistence. Finally, we use the improved cellular automata model to simulate how employee behavior evolves, and how different encouragement rules and punishment rules influence employees' behavior.

\section{References}

1. Mark John Somers: Ethical Codes of Conduct and Organizational Context: A Study of the Relationship between Codes of Conduct, Employee Behavior and Organizational Values. Journal of Business Ethics 30(2001) 185-195, 2001

2. PALASH SARKAR: A Brief History of Cellular Automata. ACM Computing Surveys 1(2000) 80-107

3. L. A. Burke, L. A. Witt: Personality and High-maintenance Employee Behavior. Journal of Business and Psychology 3(2004) 349-363

4. Dane K. Peterson: The Relationship between Unethical Behavior and the Dimensions of the Ethical Climate Questionnaire. Journal of Business Ethics 41(2002) 313-326

5. HU Bin, ZHANG De-bin: Distance Based Cellular Automata Simulation for Employee Behaviors.Systems Engineering-Theory \& Practice 2(2006) 83-96

6. Aaron Bolin, Linette Heatherly: Predictors of Employee Deviance: The Relationship between Bad Attitudes and Bad Behavior. Journal of Business and Psychology 3(2001) 405-418 